Supporting information of the article:

\title{
Comparison of Different TMAO Force Fields and Their Impact on the Folding Equilibrium of a Hydrophobic Polymer
}

Francisco Rodríguez-Ropero, ${ }^{1}$ Philipp Rötzscher and Nico F. A. van der Vegt $^{1, *}$

${ }^{1}$ Eduard-Zintl-Institut für Anorganische und Physikalische Chemie and Center of Smart Interfaces, Technische Universität Darmstadt, Alarich-

Weiss-Straße 10, 64287, Darmstadt, Germany

*E-mail: vandervegt@csi.tu-darmstadt.de 
Table of contents
Page S3
Figure S1
PMF VS Rg
Page S4
Figure S2
Preferential binding coefficients 

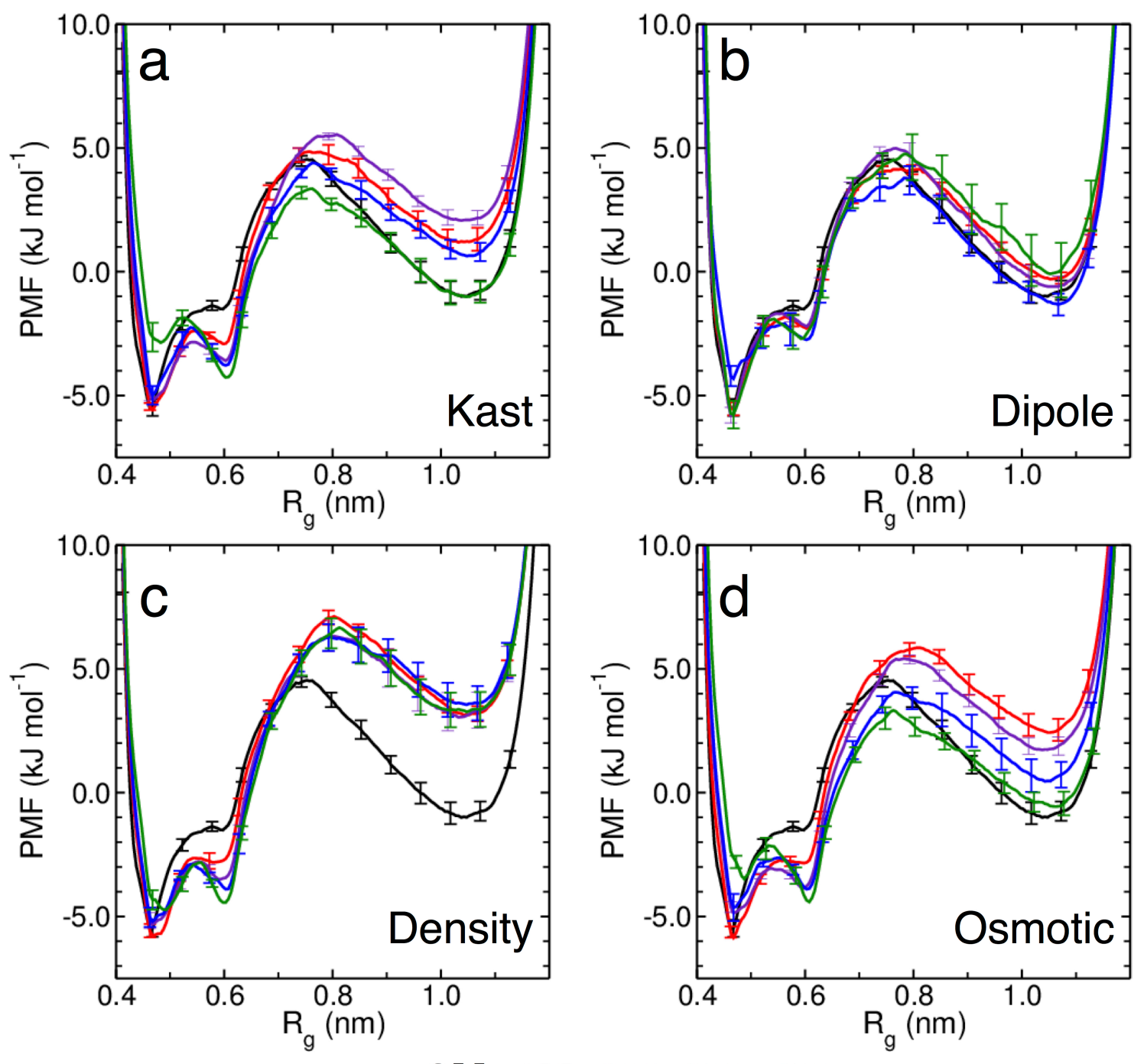

OM 1M 2M 3M 4M

Figure S1. Potentials of mean force (PMF) as a function of the radius of gyration $\left(\mathrm{R}_{\mathrm{g}}\right)$ of the hydrophobic polymer at different TMAO concentrations. 

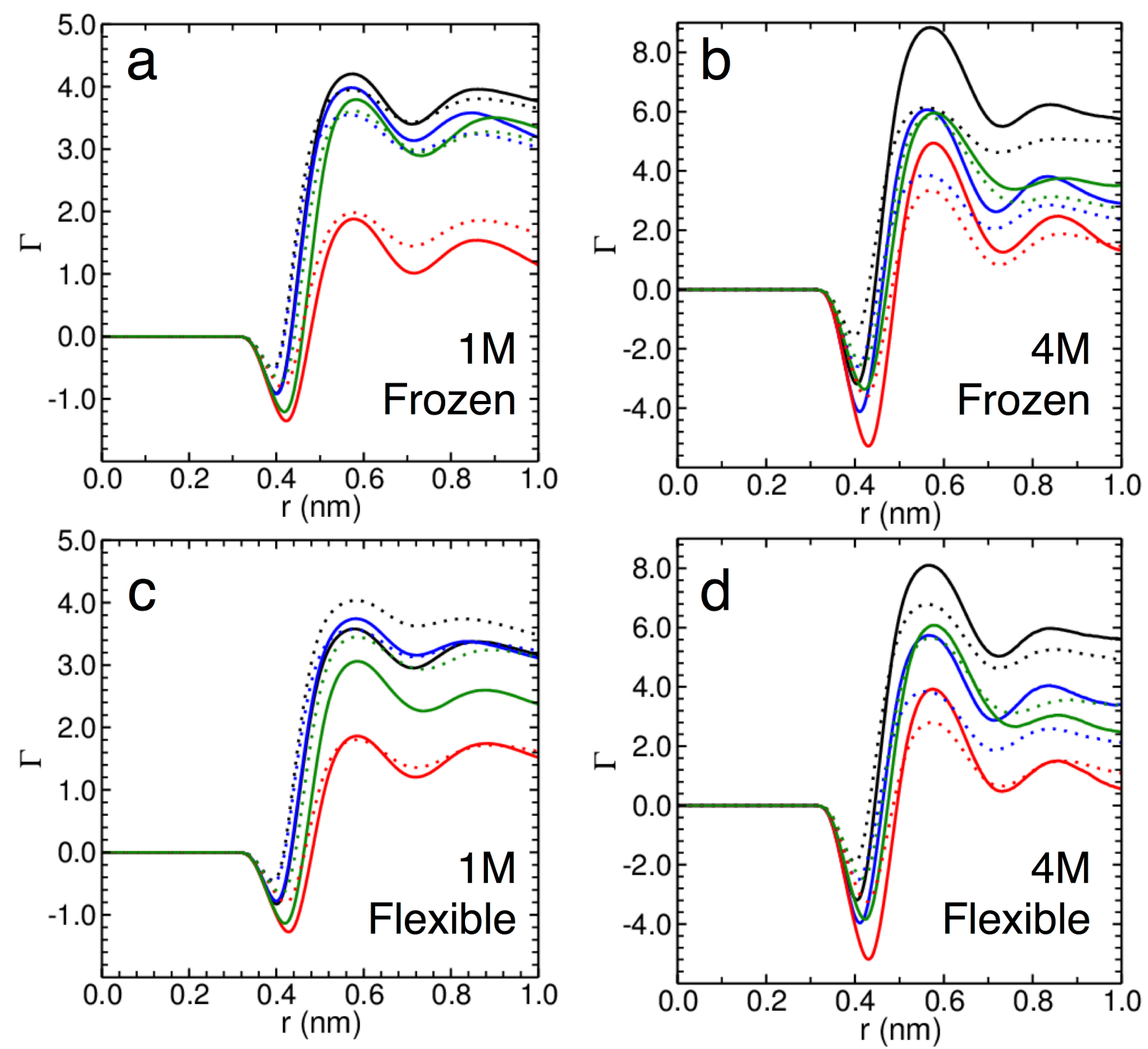

Kast Osmotic Dipole Density

Figure S2. Preferential binding coefficient $\Gamma$ for globular (dotted line) and extended (continuous line) configurations at $1 \mathrm{M}$ (left) and $4 \mathrm{M}$ (right) TMAO concentrations for frozen (left) and flexible (right) chains. 